\title{
Knockdown of the Bcl-2 gene increases sensitivity to EGFR tyrosine kinase inhibitors in the H1975 lung cancer cell line harboring T790M mutation
}

\author{
MAN ZOU, SHU XIA, LIANG ZHUANG, NA HAN, QIAN CHU, TENGFEI CHAO, \\ PING PENG, YU CHEN, QI GUI and SHIYING YU \\ Department of Oncology, Tongji Hospital, Tongji Medical College, \\ Huazhong University of Science and Technology, Wuhan 430030, P.R. China
}

Received January 30, 2013; Accepted March 20, 2013

DOI: $10.3892 /$ ijo.2013.1895

\begin{abstract}
Epidermal growth factor receptor tyrosine kinase inhibitors (EGFR-TKIs) are being widely used as targeted therapy in non-small cell lung cancer (NSCLC), but most cases acquire drug-resistance in 9 months. However, the mechanisms of resistance are still not fully understood. Since it has been demonstrated that EGFR-TKI-mediated repression of downstream signaling cascades and apoptosis induction is a key mechanism through which EGFR-TKIs exert their cytotoxic effects, we reasoned that activation of downstream signaling pathways and changes in the expression of apoptosis-related proteins contribute to the acquired resistance to EGFR-TKIs. We analyzed the protein levels of p-Akt, Bcl-2, Bax between gefitinib-sensitive and gefitinib-resistant lung cancer cell lines and evaluated whether targeting the anti-apoptotic protein Bcl-2 induces cell apoptosis and further sensitizes resistant H1975 cells to gefitinib. The data showed that p-Akt was activated and accompanied by substantial Bcl-2 in the H1975 lung cancer cell line, whereas no evidence was observed in HCC827 cells. Using small interfering RNA (siRNA) to silence Bcl-2 in H1975 cells led to significant downregulation of Bcl-2 protein expression, decreased cell viability in vitro and induced intrinsic apoptosis confirmed by flow cytometry and PARP
\end{abstract}

Correspondence to: Dr Shiying Yu, Department of Oncology, Tongji Hospital, Tongji Medical College, Huazhong University of Science and Technology, 1095 Jiefang Road, Wuhan 430030, P.R. China

E-mail: syyu@tjh.tjmu.edu.cn

Abbreviations: NSCLC, non-small cell lung cancer; SCLC, small cell lung cancer; EGFR-TKI, epidermal growth factor receptor tyrosine kinase inhibitor; PI3K, phosphoinositide 3-kinases; siRNA, small interfering RNA; FBS, fetal bovine serum; MTT, 3-(4,5-dimethylthiazol-2-yl)-2, 5-diphenyltetrazolium bromide; IR, inhibition rate; PBS, phosphate buffer saline; DAPI, 4',6-diamidino2-phenylindole; CLL, chronic lymphocytic leukemia

Key words: gefitinib, Bcl-2, RNA interference, apoptosis, lung cancer, acquired resistance cleavage. In Bcl-2 siRNA-transfected cells, adding gefitinib further reduced the number of viable cells, induced apoptosis to a greater extent compared to either treatment alone. These preclinical data suggested that downregulation of Bcl-2 by RNAi in the gefitinib-resistant H1975 lung cancer cell line with T790M mutation enhanced the effects of gefitinib and may offer a novel therapeutic strategy for the treatment of NSCLC.

\section{Introduction}

Lung cancer is the most lethal cancer in the world. Non-small cell lung cancer (NSCLC), the majority form of lung cancer, is often in advanced stage when diagnosed and patients do not have the opportunity for surgical resection. Patients did not benefit much from traditional chemotherapy and radiotherapy; therefore the 5-year survival rate remained poor (1). In recent years, along with the invention of targeted therapy, more people benefit from the treatment and survive longer.

Gefitinib is a first generation epidermal growth factor receptor tyrosine kinase inhibitor (EGFR-TKI) which prevent the phosphorylation of EGFR as well as the signaling transduction (2). Clinically, only a subtype of patients is suitable to take EGFR-TKI as the major therapy. They were mostly Asians, females, non-smokers, adenocarcinomas and had sensitive mutation in exons encoding the tyrosine kinase domain of EGFR gene $(3,4)$. However, to our disappointment, most patients could not escape a relapse when they took the drug for a median time of 9 months (5). A wide array of research has been done to explore the exact mechanism of acquired EGFR-TKI resistance. In 2005, the first case report on T790M mutation found that patient relapsed after receiving TKI drugs, Pao et al found that $50 \%$ of the cases progressed because they had the T790M mutation $(6,7)$. Another $20 \%$ of secondary resistance was discovered in 2007 because of the amplification of the c-Met gene (8). Still, the remaining $30 \%$ are extensively investigated, for loss of PTEN gene, loss of IGF-binding protein, expression of epithelial membrane protein-1 (EMP-1), and activation of downstream EGFR signaling (9-13). Shaw et al reported in 2009 that in NSCLC patients with metastatic disease, who harbored EML4-ALK mutation, would become resistant to EGFR-TKI (14). However, the majority of extensive pre-clinical studies 
involving in vitro and in vivo experiments have not discovered new drug candidate having scientific merit for further development. Thus, we explored the untapped mechanism of acquired gefitinib resistance so as to provide a combinatorial strategy in H1975 gefitinib-resistant cell line using gefitinib as a main compound.

P-Akt was found elevated in many tumor tissue samples and cancer cell lines, including esophageal cancer, lung cancer and its expression is often related to poor clinical outcome and indicate the resistance of therapy (15-17). Since phosphoinositide 3-kinases (PI3K)/Akt pathway is vital in tumor development and progression, once activated, it would affect several downstream signaling pathways, such as proliferation, survival, anti-apoptotic, cell cycle and angiogenesis $(18,19)$. Targeting the constitutively active Akt kinase and its key downstream molecules might provide a possible anticancer effect. Synergistic use of chemotherapy drugs with mTOR inhibitor exhibited great inhibition influence on renal cell carcinoma and was approved by FDA for use in patients with metastatic renal cell carcinoma (20). However, due to complicated interactions or compensatory upregulation of activated Akt, combining EGFR-TKI with mTOR inhibitor in lung cancer failed to reach the ideal result either in laboratory or in the latest clinical trials $(21,22)$. It is, therefore, important to discover novel alternative Akt downstream targets which do not cause a negative feedback loop, allowing to reverse therapeutic resistance to TKI drugs. In 2004, Brown et al had found reduced expression of p27 was a novel mechanism of docetaxel resistance in breast cancer cells (23). Similarly, the downstream molecule Bim of Akt signaling is also involved in mediating EGFR-TKI-induced apoptosis in lung cancer cells (24). Knockdown of Bim was able to attenuate apoptosis induced by EGFR-TKI (24).

It is well known that gefitinib induces apoptosis in NSCLC cell line H3255 (2). Consequently, the development of resistance of cancer cells to TKI drugs may be a result of resistance to apoptosis. Bcl-2 is an Akt downstream anti-apoptotic protein belonging to the Bcl-2 family. In many tumor cells, such as breast cancer, and chronic lymphocytic leukemia, the knockdown Bcl-2 expression can induce cell apoptosis and even overcome drug resistance $(25,26)$. We wondered whether gefitinib resistance was associated with the dysregulation of apoptosis in H1975 cell line. RNA interference is a more effective method to silence gene expression from RNA level to protein level compared with antisense method (27). We examined the influence of Bcl-2 small interfering RNA (siRNA) on the drug sensitization in H1975 cells. Our results revealed that the knockdown Bcl-2 gene expression by siRNA induced cell apoptosis in H1975 lung cancer cell line. Furthermore, gefitinib enhanced pro-apoptotic effect and reversed acquired EGFR-TKI drug resistance. Our data predicted a potential of a combined therapeutic approach with gefitinib and $\mathrm{Bcl}-2$ siRNA for the treatment of EGFR-TKI-resistant H1975 cell line containing T790M mutation.

\section{Materials and methods}

Materials and reagents. The following monoclonal antibodies were used: Akt (Epitomics, CA, USA), p-Akt (Cell Signaling Technology, Danvers, MA, USA), Bcl-2 (Epitomics), Bax
(Epitomics), caspase-3 (Epitomics),PARP-p110/85 (Epitomics), GAPDH (Epitomics). Goat anti-rabbit peroxidase-conjugated secondary antibody (Boster, Wuhan, China). The sequence of siRNA targeting Bcl-2 were: si-h-BCL2_001 sense, 5'-CGG AGGCUGGGAUGCCUUUdTdT-3'; antisense, 3'-dTdTGC CUCCGACCCUACGGAAA-5'; si-h-BCL2_002 sense, 5'-GG AUUGUGGCCUUCUUUGA dTdT-3'; antisense, 3'-dTdTCC UAACACCGGAAGAAACU-5'; si-h-BCL2_003 sense, 5'-GG AUGACUGAGUACCUGAA dTdT-3'; antisense, 3'-dTdT CCUACUGACUCAUGGACUU-5'. SiRNA against Bcl-2 and a negative control siRNA were purchased from Guangzhou RiboBio Co. Ltd. (Guangzhou, China). Lipofectamine 2000 was obtained from Invitrogen (Carlsbad, CA, USA). Gefitinib was generously provided by AstraZeneca and was dissolved in DMSO in $20-\mathrm{mM}$ concentration stored at $-20^{\circ} \mathrm{C}$. Drugs were diluted in fresh media immediately prior to use and the final DMSO concentration was $<0.1 \%$. MTT cell viability assay kit was obtained from Sigma-Aldrich (St. Louis, MO, USA). DAPI was purchased from Beyotime Institute of Biotechnology (Shanghai, China). Annexin V-FITC apoptosis detection kit was obtained from KeyGen Biotech Co. (Nanjing, China).

Cell culture. The human lung cancer H1975 and HCC827 cell lines were obtained from the Cell Bank of the Chinese Academy of Sciences (Shanghai, China). H1975 cell line was cultivated in DMEM high-glucose medium (Gibco, Grand Island, NY, USA) supplemented with $10 \%$ fetal bovine serum (FBS) (Gibco), HCC827 cell line was grown in RPMI-1640 medium (Gibco) supplemented with $10 \%$ fetal bovine serum (Gibco). Cells were incubated in a humidified incubator at $37^{\circ} \mathrm{C}$ with $5 \% \mathrm{CO}_{2}$ atmosphere and maintained in a logarithmic growth phase for all the experiments.

Transient transfection. To study the uptake of cy3-conjugated scrambled negative control siRNA, after plating $2.5 \times 10^{4}$ cells/ well in 24-well plates, transfection efficiency of siRNA at various concentrations was observed by fluorescence microscopy. We chose $50 \mathrm{nM}$ which shows $>80 \%$ transfection efficiency in the following experiments. For confirmation of downregulation of $\mathrm{Bcl}-2$ protein, three siRNA oligonucleotides directed against $\mathrm{Bcl}-2$ and a negative control siRNA were tested for effectiveness of protein knockdown. In brief, $1 \times 10^{5}$ cells were plated in 6-well plates, incubated for $24 \mathrm{~h}$ and transfected with siRNA at $50 \mathrm{nM}$ using Lipofectamine 2000 reagent and OPTI-MEM reduced serum media (Gibco) according to the manufacturer's instructions. Later (4-6 h), OPTI-MEM media was aspirated and DMEM fresh media containing 10\% FBS was added. At $48 \mathrm{~h}$ after transfection, cellular expression of Bcl-2 was determined by western blot analysis.

MTT assay. Cell viability assay was performed with MTT cell viability assay kit. Cells were seeded into 5 replicate wells of each group, at a density of $6 \times 10^{3}$ viable cell per well in 96-well plates. Allowed to attach for $24 \mathrm{~h}$, transfection was done. Forty-eight hours later, various concentrations of gefitinib were added and cells were incubated for another $24 \mathrm{~h}$. At the indicated time, 3-(4,5-dimethylthiazol-2-yl)-2, 5-diphenyltetrazolium bromide (MTT) was added to incubate for $4 \mathrm{~h}$. Then media were aspirated, $100 \mu \mathrm{l}$ DMSO was added. Cells were incubated for $10 \mathrm{~min}$ at $37^{\circ} \mathrm{C}$ with gentle shaking. The 
absorbance was then read at $490 \mathrm{~nm}$ with a 96-well microplate reader (Bio-Rad). The values in the siRNA treated cells were normalized to the values of control as to determine the percentage of viability. Each assay was performed in triplicate. The inhibition rate (IR) was calculated according to equation: $\mathrm{IR}=[\mathrm{A} 490$ (control) - A490 (treatment)]/[A490 (control) A490 (zero)] x 100\%.

A490 (control) stands for the absorbance in control group. A490 (treatment) was the absorbance in the drug-treated or siRNA groups. A490 (zero) means the absorbance in the group with no cells.

DAPI staining. Cells were seeded into 24-well plates, at a density of $2 \times 10^{4}$ viable cells per well. After $48 \mathrm{~h}$ of transfection, cells were treated with $5 \mu \mathrm{mol} / 1$ gefitinib. Drug dose was based on the $\mathrm{IC}_{50}$ data in the cell viability assay and available data from other studies (28). Following culture for further $24 \mathrm{~h}$, the cells were washed with phosphate buffer saline (PBS) and fixed with $4 \%$ paraformaldehyde, then washed with PBS 3 times. Cells were stained with 4',6-diamidino-2-phenylindole (DAPI) for $10 \mathrm{~min}$ and washed another 3 times. Cell nuclear morphology was examined by UV fluorescent microscopy. Apoptotic cells were identified by condensation and fragmentation of nuclear chromosome. At least 200 cells were counted and performed in a blinded manner.

Apoptosis detection. Cells $\left(1 \times 10^{5}\right)$ were plated in 6-well plates in $2 \mathrm{ml}$ of fresh media and cultured for $24 \mathrm{~h}$. After H1975 cells had been transfected with Bcl-2 siRNA or negative siRNA for $48 \mathrm{~h}$, cells were left untreated or exposed to gefitinib at a concentration of $5 \mu \mathrm{mol} / \mathrm{l}$. Twenty-four hours later, cells attached to the growth surface were removed by trypsin treatment [trypsin $\left.(0.05 \%) ; 37^{\circ} \mathrm{C}, 1 \mathrm{~min}\right]$. Attached and detached cells were collected for analysis, washed twice in cold PBS and resuspended in $500 \mu \mathrm{l}$ of binding buffer containing $5 \mu \mathrm{l}$ Annexin-V-FITC and $5 \mu \mathrm{l}$ propidium iodide mixtures according to the manufacturer's instructions. FACS analysis was performed on FACScan (Becton-Dickinson Co., USA) using CellQuest software (Becton-Dickinson), 10,000 events were collected for each sample.

Protein extraction and immunoblotting. Cells were collected, washed twice in ice-cold PBS and lysed in ice-cold lysis buffer containing $20 \mathrm{mMTris}$ (pH 7.5), $150 \mathrm{mMNaCl}, 1 \%$ Triton X-100, $2.5 \mathrm{mM}$ sodium pyrophosphate, $1 \mathrm{mM} \beta$-glycerophosphate, $1 \mathrm{mM}$ EDTA, $1 \mathrm{mM} \mathrm{Na} \mathrm{VO}_{4}, 1 \mu \mathrm{g} / \mathrm{ml}$ leupeptin with added $1 \mathrm{mM}$ PMSF and $1 \mathrm{mM}$ phosphatase inhibitor. Centrifuged at $13,000 \mathrm{~g}$ for $5 \mathrm{~min}$ at $4^{\circ} \mathrm{C}$ and the supernatant was saved and boiled with loading buffer for $5 \mathrm{~min}$ then stored at $-80^{\circ} \mathrm{C}$ for subsequent analysis. Protein concentration was determined using BCA protein assay kit (Beyotime, Shanghai, China). Equal amounts of proteins $(40 \mu \mathrm{g})$ were loaded onto $10-12 \%$ SDS-PAGE, separated by electrophoresis and transferred to PVDF membrane (Millipore, USA). Membranes were then blocked with $5 \%$ non-fat milk containing $0.1 \%$ Tween-20 at room temperature for $1 \mathrm{~h}$, incubated with primary antibodies [p-Akt (1:2,000); Akt (1:10,000); Bcl-2 (1:500); Bax (1:1,000); caspase-3 (1:1,000); PARP p110/85 (1:1,000); GAPDH $(1: 10,000) ; 4^{\circ} \mathrm{C}$, overnight] and subsequently with goat antirabbit peroxidase-conjugated secondary antibody (1:2,000; room temperature, $1 \mathrm{~h}$ ). Peroxidase activity was visualized with Pierce Super Signal West Pico Chemiluminescent Substrate (Pierce, USA). Signal intensity was determined densitometrically and normalized against those of total proteins present in the corresponding lane on the membrane using Quality one software, version 1.5 (Bio-Rad, CA, USA).

Statistical analysis. Statistical analysis was carried out using SPSS software, version 17.0 (Chicago, IL, USA). Data are presented as mean \pm SD from at least three experiments. Results were analyzed using Student's t-test and p-values were indicated where appropriate in the figures and in their legends. A $p<0.05$ was considered statistically significant.

\section{Results}

Gefitinib-induced cytotoxicity is substantially reduced in gefitinib-resistant H1975 cell line compared with gefitinibsensitive HCC827 cell line. Lung cancer H1975 cell line is an established cell line bearing T790M mutation besides L858R mutation and is being used as a model for acquired resistance to EGFR-TKI. In this experiment, we chose HCC827 lung cancer cell line containing exon 19 deletion as a sensitive control. To measure the growth inhibitory action of geifitinib and to confirm each cell line as either sensitive or resistant, we performed MTT assays. For each cell line, cells were incubated for $\leq 48 \mathrm{~h}$ at indicated doses of gefitinib $(0,0.001,0.01,0.1,1$, 10 or $100 \mu \mathrm{mol} / \mathrm{l}$ ). The results (Fig. 1A and B) demonstrated that gefitinib exhibited time- and dose-dependent inhibition effect on HCC827 cell line, and slightly inhibited the proliferation of H1975 cells. After 24-h exposure to gefitinib, H1975 gefitinib-resistant subtype exhibited greater resistance to gefitinib than did HCC827 cells $\left(\mathrm{IC}_{50} 35.75 \pm 0.77 \mu \mathrm{mol} / \mathrm{l}\right.$ vs $0.003 \pm 0.18 \mu \mathrm{mol} / 1$, respectively).

Akt activation and downstream protein expression in different cell lines. To test whether PI3K/Akt signaling cascade activation exists in cells with acquired resistance to gefitinib, we used immunoblotting to test expression of several proteins between gefitinib-resistant and gefitinib-sensitive cell lines. As expected, phosphorylated Akt was elevated in H1975 cell line compared with HCC827 cell line (Fig. 1C). Since we and others had found that gefitinib induce apoptosis and downregulate phosphorylated Akt expression in HCC827 cell line, we presumed that acquired gefitinib resistance might be due to the overexpression of certain inhibitor proteins of apoptosis which belong to the Akt downstream of signaling cascade (2). We compared the basic level of Bcl-2 family apoptosis-related proteins in two cell lines. Indeed, we found abundant antiapoptotic Bcl-2 protein in H1975 cells, whereas no evidence was detected in HCC827 cells (Fig. 1C). There was no significant difference in the expression of pro-apoptotic Bcl-2 associated X protein (Bax) (Fig. 1C).

Knockdown of the Bcl-2 gene in H1975 lung cancer cell line. Based on the above results, we performed RNA interference methodology to downregulate Bcl-2 protein expression in H1975 cell line. We used cy3-conjugated siRNA to verify the transfection efficiency though fluorescence microscopy, the transfection efficiency in this cell line can reach $>80 \%$ when 
A

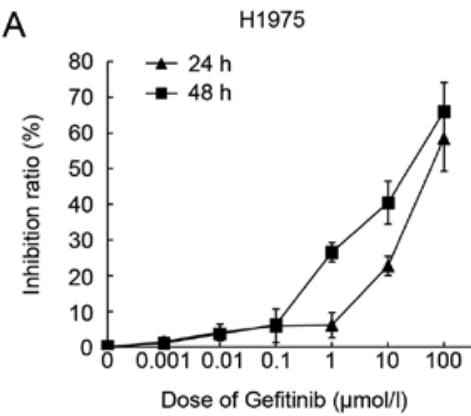

B
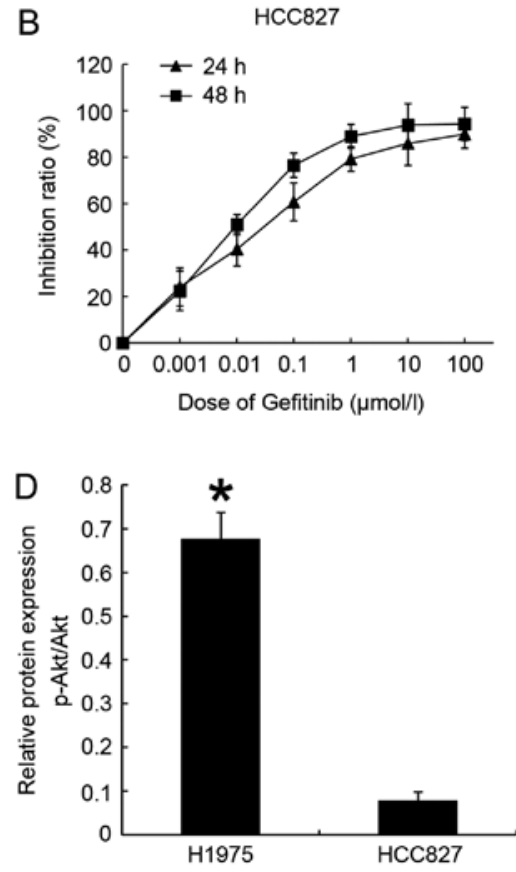
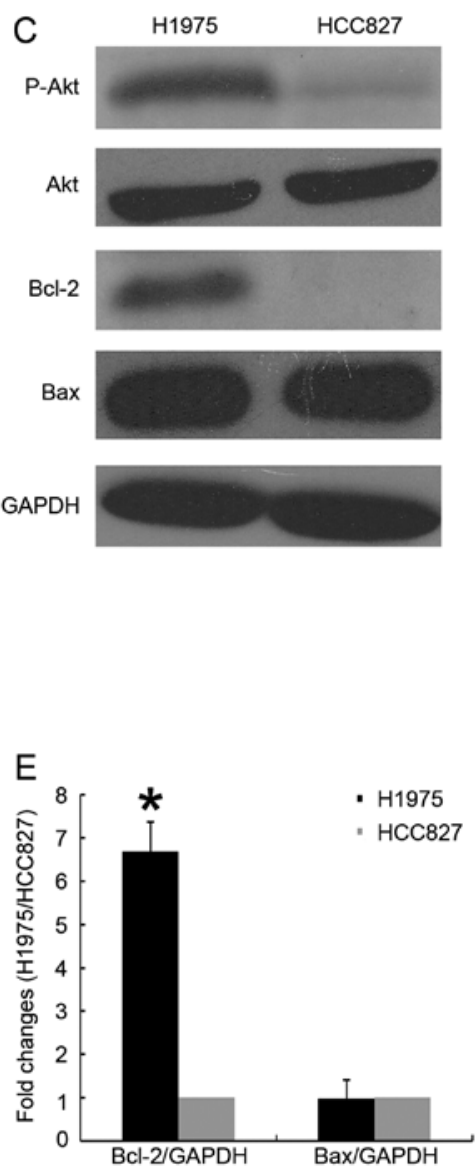

Figure 1. Gefitinib inhibits cell proliferation and protein expression distinction in two NSCLC cell lines. (A and B) Gefitinib inhibited cell growth in both H1975 and HCC827 cell lines in a dose- and time-dependent manner. Results are the mean of three independent experiments (each with five replicates), (C) Total cell protein extracts was analysed by western blotting to detect p-Akt, Akt, Bcl-2 and Bax protein expression in NSCLC H1975 and HCC827 cell lines. GAPDH was used as the control. (D) Analysis of relative p-Akt protein expression in two cell lines. (E) Fold changes of Bcl-2/GAPDH and bax/GAPDH in $\mathrm{H} 1975$ cells and HCC827 cells. The histogram shows the mean \pm SD of three individual studies. " $p<0.05$ compared to HCC 827 cells.

siRNA concentration is $50 \mathrm{nM}$. After $48 \mathrm{~h}$ of transfection, the effect of siRNA on the protein level was assessed (Fig. 2A). A small amount of Bcl-2 in transfected group was detected from the immunoblotting. The suppression lasted for $72 \mathrm{~h}$ post-transfection. We chose siRNA-Bcl-2_003 as the most efficacious one for the following experiments (Fig. 2A). No Bcl-2 downregulation was detected in the negative siRNA group or in mock transfected cells (Fig. 2B), and, expression of protein Bax had not changed (Fig. 2B), indicating a specific and effective function, thus excluding that the effects were due to the upregulated Bax.

Bcl-2 siRNA increases gefitinib sensitivity. We examined the effects of siRNA mediated silencing of Bcl-2 protein expression on the proliferation of $\mathrm{H} 1975$ lung adenocarcinoma cells. After being transfected with Bcl-2 siRNA for $48 \mathrm{~h}$, viable cells were reduced to $58.1 \%$ compared with the untreated control group (Fig. 3A). Although negative siRNA group and mock transfected cells exhibited minor killing effect, there was no statistical significance ( $p>0.05)$. The results showed that downregulating Bcl-2 expression was able to restore the effect of gefitinib in H1975 cells, Bcl-2 siRNA transfectants had higher IR than negative siRNA transfectants or mock cells $(\mathrm{p}<0.05)$. After a 48 -h period allowing silencing of Bcl-2, H1975 cells were further exposed to a series concentrations of gefitinib $(0,0.5,1,5,10,50$ or $100 \mu \mathrm{mol} / \mathrm{l})$ for $24 \mathrm{~h}$. As shown in Fig. 3B, we observed that combination treatment significantly augmented IR 4.6-fold compared with control group with gefitinib $\left(\mathrm{IC}_{50} 7.07 \pm 0.38\right.$ vs $32.47 \pm 0.71 \mu \mathrm{mol} / 1$, respectively) and 4.3 -fold compared with negative siRNA plus gefitinib $\left(\mathrm{IC}_{50} 7.07 \pm 0.38\right.$ vs $30.27 \pm 0.61 \mu \mathrm{mol} / 1$, respectively). Results indicated that combining Bcl-2 siRNA and gefitinib induced synergistic inhibitory effect on H1975 cell proliferation and viability.

Effect of Bcl-2 siRNA on the nuclear morphology change. We investigated whether the anti-proliferation effect of Bcl-2 siRNA in H1975 cells was due to the induction of apoptosis, and whether the knockdown of Bcl-2 might sensitize the cells to undergo apoptosis in response to gefitinib. To provide 
A

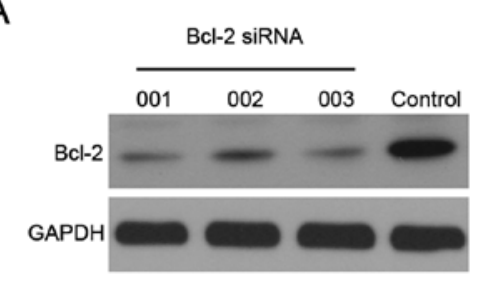

C

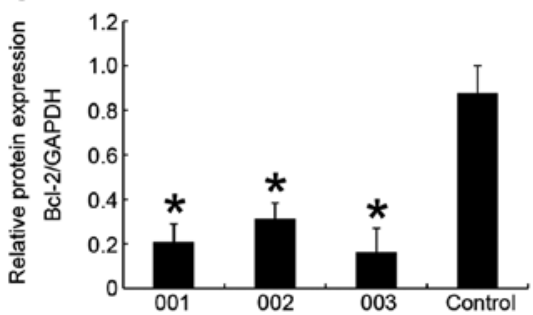

B

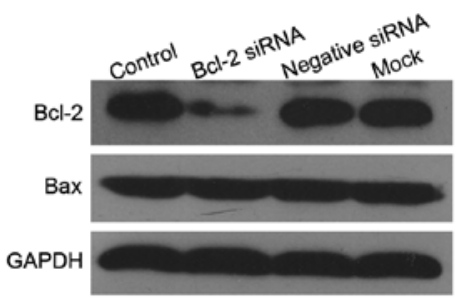

D

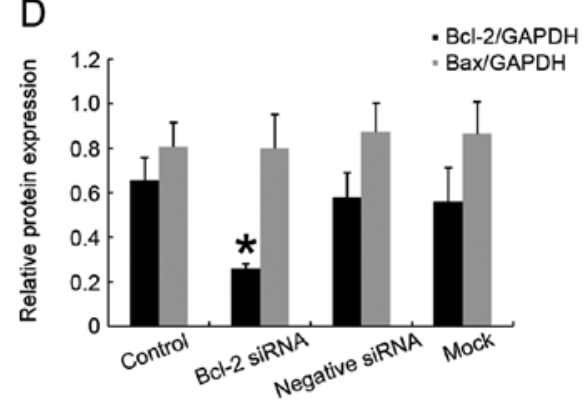

Figure 2. Effects of Bcl-2 small interfering RNA on Bcl-2 protein expression in H1975 cell line. (A) Comparison of the silencing effect of Bcl-2 siRNA001, Bcl-2 siRNA002 and Bcl-2 siRNA003 on NSCLC H1975 cell line. Cells were transfected with $50 \mathrm{nM} \mathrm{Bcl-2} \mathrm{siRNAs} \mathrm{and} 48 \mathrm{~h}$ post transfection, lysed and $40-\mu \mathrm{g}$ proteins was used for detection. (B) Specific inhibition of Bcl-2 protein expression was tested. Cells without treatment (control), negative siRNA transfected (negative siRNA), mock transfected (mock, all regeants except for siRNA) were used as controls. (C) Analysis of protein level of each Bcl-2 siRNA group with untreated control group. (D) Bcl-2 protein expression was significantly decreased in Bcl-2 siRNA003-treated group while no distinctive change was observed in other control groups. Bcl-2 siRNA does not affect the expression of Bax protein. GAPDH was used as the internal control. The histogram shows the mean $\pm \mathrm{SD}$ for triplicate analysis. ${ }^{*} \mathrm{p}<0.05$ vs. control group.
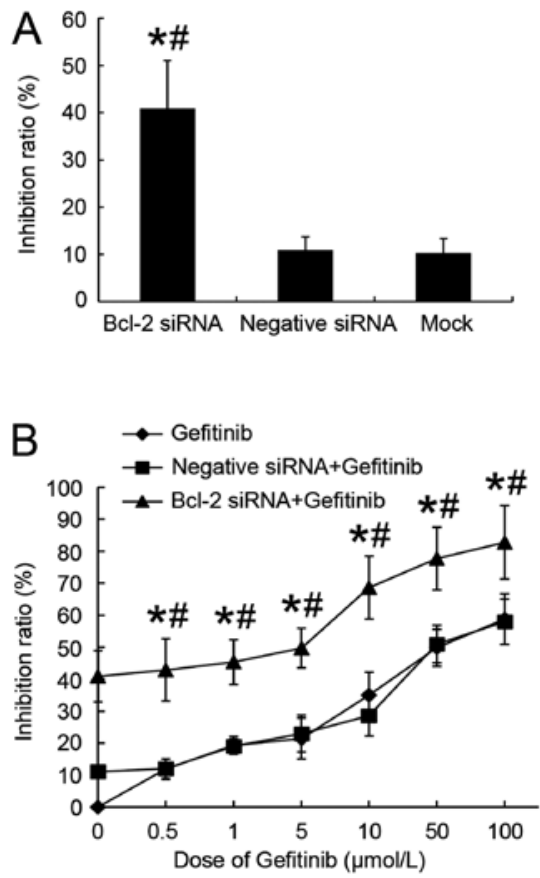

Figure 3. Bcl-2 siRNA inhibits cell proliferation and sensitizes H1975 cell line to gefitinib. (A) Cells were incubated with $50 \mathrm{nM} \mathrm{Bcl-2}$ siRNA, negative siRNA or only Lipofectamine 2000 for $48 \mathrm{~h}$. MTT assay was used to determine the cell proliferation inhibition rate. "p $<0.05$ vs. negative siRNA group while ${ }^{\#} p<0.05$ vs. mock group. (B) Cells were incubated with different doses of gefitinib for $24 \mathrm{~h}$ after being transfected with Bcl-2 siRNA or not. " $\mathrm{p}<0.05$ vs. negative siRNA with gefitinib while ${ }^{\#} \mathrm{p}<0.05$ vs. gefitinib only group.

confirmatory evidence, DAPI staining was performed to detect nuclear morphological changes of the H1975 cells being transiently transfected in the presence or absence of gefitinib.
We chose $5 \mu \mathrm{mol} / 1$ according to the $\mathrm{IC}_{50}$ and also because this is a concentration that can be achieved in serum of patients being treated with gefitinib for short period (29). As shown in Fig. 4A, cells transfected with siRNA targeting Bcl-2 for $48 \mathrm{~h}$ underwent characteristics of apoptosis, including chromatin condensation and nuclear DNA fragmentation. When followed by $5 \mu \mathrm{mol} / 1$ gefitinib administration for $24 \mathrm{~h}$, the nuclear change became more obvious. Inversely, untreated control cells, merely suffering higher dose of gefitinib $(20 \mu \mathrm{mol} / \mathrm{l})$ and cells transfected with negative siRNA all showed only slight signs of apoptosis.

Effect of Bcl-2 siRNA on cellular apoptosis. To further investigate the exactly apoptosis rate among different groups, we performed flow cytometry. The Annexin-V-FITC assay was aimed to distinguish whether the reduction in cell viability was due to apoptosis or necrosis. As seen in Fig. 4B, after Bcl-2 siRNA treatment, the rate of early apoptotic cells increased from 7.98 in control group to $21.35 \%$. This difference is statistically significant $(\mathrm{p}<0.05)$. The percentage of late apoptotic cells was also augmented. To determine whether the time to add gefitinib impacted on the synergistic effect, we evaluated different groups according to the time-points that gefitinib was added. Cells were treated with $5 \mu \mathrm{mol} / 1$ gefitinib at the time of transfection end, $24 \mathrm{~h}$ later or $48 \mathrm{~h}$ after transfection. When followed by $5 \mu \mathrm{mol} / \mathrm{l}$ gefitinib addition $48 \mathrm{~h}$ after transfection, the early apoptotic fraction increased to a greater extent, from 21.35 in the absence to $29.27 \%$ in the presence. Our results showed only the group that added gefitinib $\geq 48 \mathrm{~h}$ after transfection had synergistic function. This synergistic effect could not be seen in other groups when gefitinib was added at an earlier time either immediately after transfection or $24 \mathrm{~h}$ later. Results above suggested that knockdown of 
A

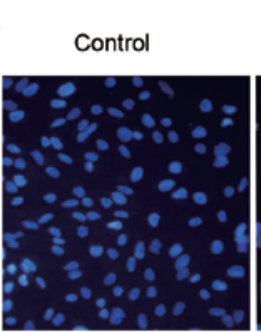

Gefitinib
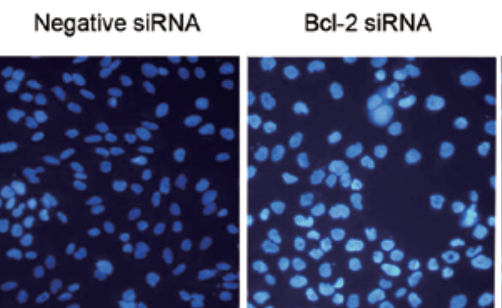

Bcl-2 siRNA+Gefitinib

B
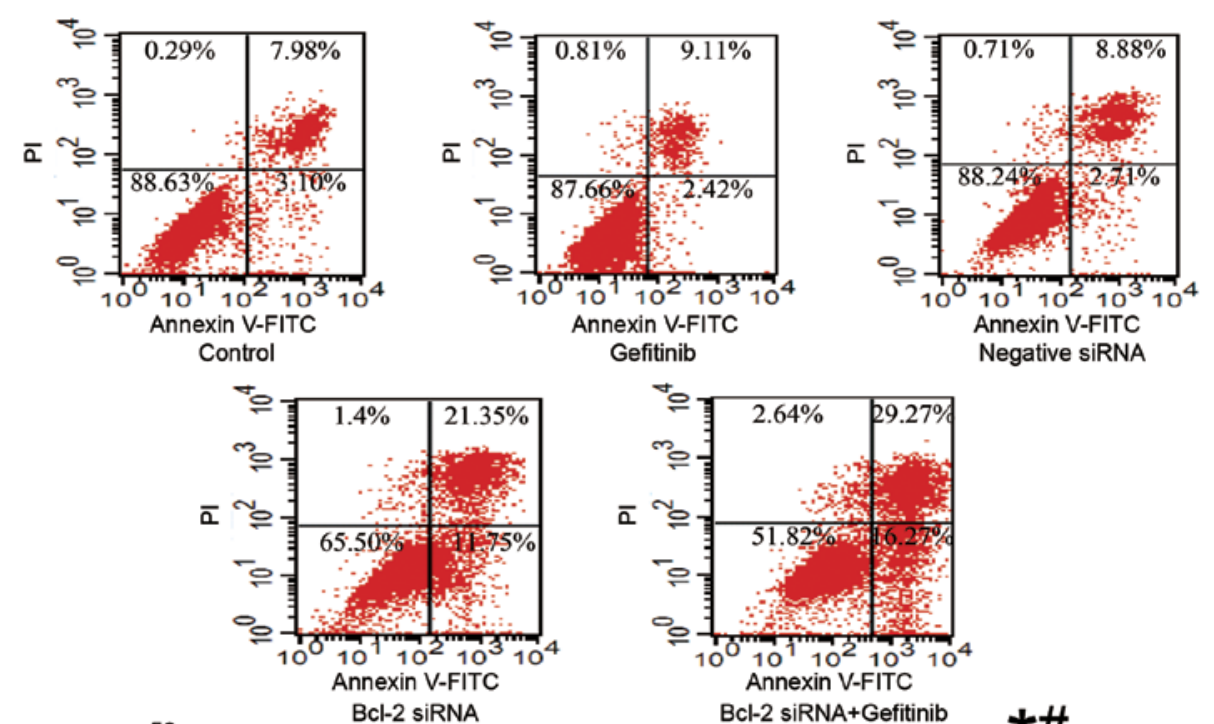

Gefitinib

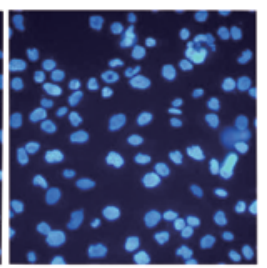

C

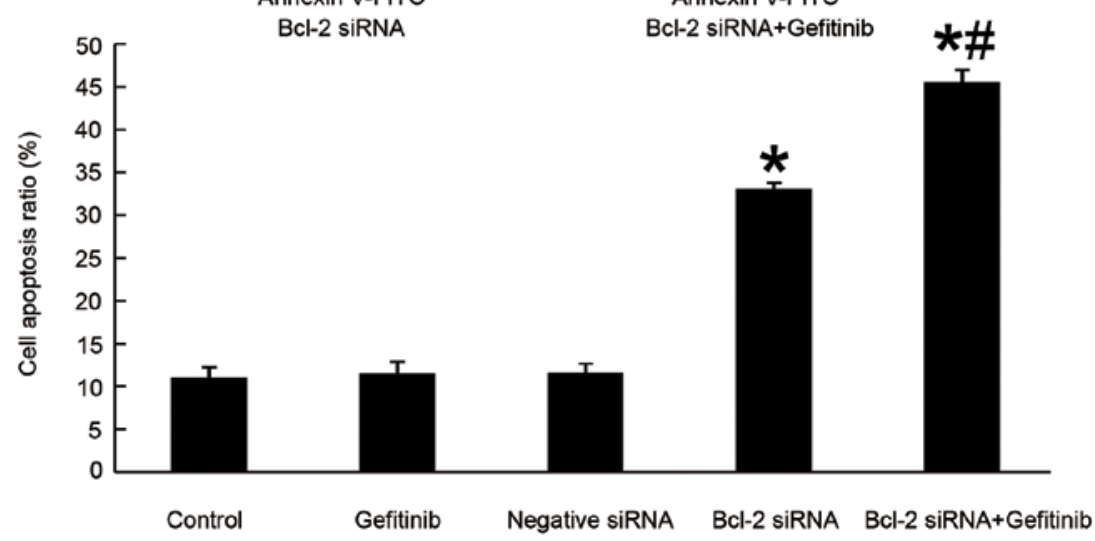

Figure 4. Cell nuclear morphological change and determination of apoptosis rate after downregulation of Bcl-2 expression in H1975 cell line in the presence or absence of gefitinib. (A) Nuclear morphology of H1975 cells were stained with DAPI. Images from left to right: cells without treatment (control); cells with $20 \mu \mathrm{mol} / 1$ gefitinib for $24 \mathrm{~h}$ (gefitinib); negative siRNA transfectants (negative siRNA); Bcl-2 siRNA transfectants (Bcl-2 siRNA); Bcl-2 siRNA transfected followed by $5 \mu \mathrm{mol} / 1$ gefitinib for $24 \mathrm{~h}$ (Bcl-2 siRNA+gefitinib). Upon treatment with Bcl-2 siRNA, cells showed characteristic apoptotic morphology of nuclear DNA condensation, marginalization or fragmentation while no observable change could be seen from negative siRNA group or untreated group. When followed by $5 \mu \mathrm{mol} / 1$ gefitinib administration for $24 \mathrm{~h}$, the nuclear change became more significant (magnification, x200). (B) Flow cytometry analysis using Annexin V-FITC and propidium iodide double staining was performed to detect the apoptosis ratio. Cells transfected with Bcl-2 siRNA induced apoptosis and was further enhanced by combination of Bcl-2 siRNA plus gefitinib. (C) Comparison of the total apoptosis rate including early and late apoptosis populations. Early apoptotic cells were positive for Annexin V-FITC and negative for PI. Late apoptotic cells were positive for both Annexin V-FITC and PI. The data are presented as means \pm SD for triplicate analysis per group. ${ }^{*} p<0.05$ vs. control group and ${ }^{*} p<0.05$ vs. Bcl- 2 siRNA group.

Bcl-2 protein expression in H1975 lung cancer cell line can suppress cell proliferation via apoptosis induction. It also demonstrated that synergy between Bcl-2 siRNA and gefitinib was augmented by optimizing drug scheduling with superior effects when gefitinib was administered $48 \mathrm{~h}$ after transfection at the time that Bcl-2 had been successfully knocked down. The data coincided with the hypothesis that Bcl-2 expression level is associated to the sensitivity of EGFR-TKI drugs.

Effect of Bcl-2 siRNA activated caspase-3 and increased PARP cleavage through regulating intrinsic mitochondrial pathways of apoptosis. The pro-apoptotic effect of Bcl-2 siRNA was further confirmed by examining the total and cleavage protein of caspase- 3 and its downstream substrate PARP. As seen in Fig. 5A once cells were treated with Bcl-2 siRNA will activate procaspase-3 then induce $17-\mathrm{kDa}$ active caspase-3, PARP was cleaved from its $116-\mathrm{kDa}$ intact form into $85-\mathrm{kDa}$ cleaved fragment. Cleaved PARP which is a marker of caspase-mediated early apoptosis was increased in Bcl-2 siRNA treated cells and those followed by gefitinib treatment after transfection (30). Inversely, not much cleaved PARP could be seen in the other groups. From the experiment, we observed onset of the Bcl-2 

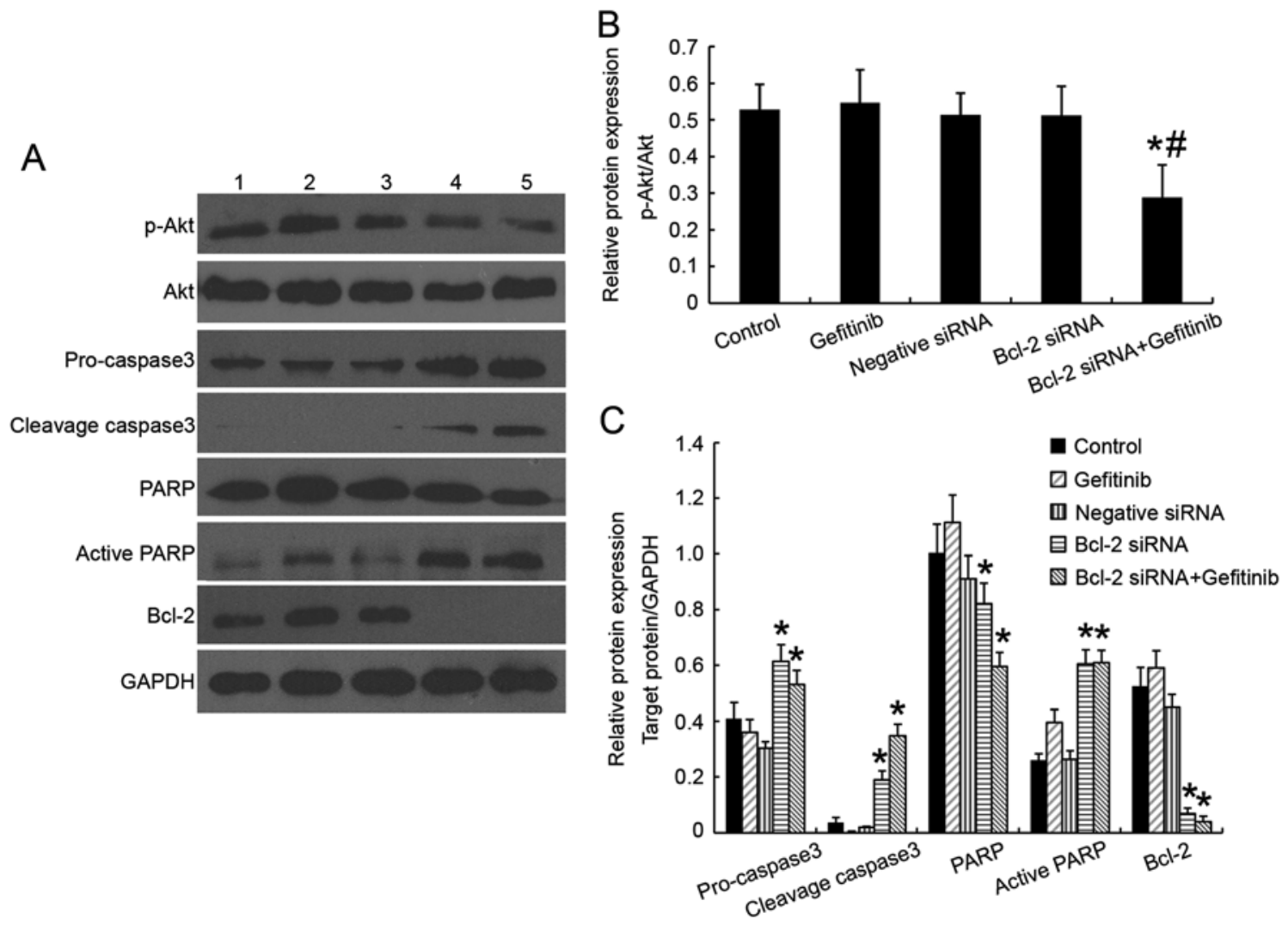

Figure 5. Effects of Bcl-2 siRNA with or without gefitinib on Akt activation and apoptosis protein expression in H1975 cell line. (A) Western blotting was used to detect p-Akt, Akt, caspase-3, PARP and Bcl-2 protein expression. Lane 1, cells without treatment (control); lane 2, cells with $20 \mu \mathrm{mol} / 1 \mathrm{gefitinib}$ for $24 \mathrm{~h}$ (gefitinib); lane 3, negative siRNA transfectants (negative siRNA); lane 4, Bcl-2 siRNA transfectants (Bcl-2 siRNA); lane 5, Bcl-2 siRNA transfected followed by $5 \mu \mathrm{mol} / 1$ gefitinib for $24 \mathrm{~h}$ (Bcl-2 siRNA+gefitinib). Bcl-2 protein expression was remarkably reduced in Bcl-2 siRNA transfected groups. Active caspase-3 and active PARP was increased in Bcl-2 siRNA transfected groups. Phosphorylation of Akt decreased only when Bcl-2 siRNA was combined with incubation of $5 \mu \mathrm{mol} / 1$ gefitinib for $24 \mathrm{~h}$. (B) Relative protein expression level of p-Akt compared with total Akt. "p $<0.05$ vs. control group while " $\mathrm{p}<0.05 \mathrm{vs}$. Bcl-2 siRNA group. (C) Relative protein expression level of pro-caspase-3, cleaved caspase-3, PARP, cleaved PARP and Bcl-2 compared with GAPDH. " group. The experiments were carried out in triplicate and representative data are shown.

siRNA process of intrinsic mitochondrial way of caspasedependent apoptosis which was consistent with the finding in other cell lines $(31,32)$. We found that gefitinib alone and Bcl-2 siRNA alone did not inhibit or stimulate Akt phosphorylation in $\mathrm{H} 1975$ cell line which indicated that no direct feedback loop exists between Bcl-2 and p-Akt; however, when siRNA targeting Bcl-2 followed by addition of $5 \mu \mathrm{mol} / 1$ gefitinib, the phosphorylation of Akt was reduced while the amount of total Akt expressed was consistent with the treatment results of Bcl-2 siRNA or gefitinib.

\section{Discussion}

Activated Akt expression manifests poor clinical outcome and confer chemotherapy and radiotherapy resistance in many cancer types including NSCLC, and neuronal cells $(16,33)$. Consistent with previous literature, our results show that compared with TKI-sensitive HCC827 lung cancer cell line, the level of p-Akt in TKI-resistant H1975 lung cancer cell line was elevated. In the former studies, it was found that combining EGFR-TKI with specific inhibitors of the Ras or PI3K pathways exerted different effects in several lung cancer cell types, which might correlate with the diverse expression of downstream proteins (34). Compared with LY294002 which is a potent inhibitor of PI3Ks, bortezomib, a proteasome inhibitor used in an established EGFR-TKI-resistant cell line, induced a significant inhibition of cancer cell growth and increased apoptosis suggesting that besides interfering with Akt signaling, other molecular mechanisms were involved in overcoming resistance to anti-EGFR therapies (35).

Deregulation of the Akt-dependent pathway has been well documented in a variety of human tumors $(23,24)$. We were eager to know whether the activation of Akt and its downstream cascade differs in NSCLC cell lines with different response to EGFR-TKI. We focused on apoptosis pathway downstream of Akt aiming to avoid the negative feedback induced by mTOR inhibition (36). We postulated that tyrosine kinase inhibitor resistance in lung cancer H1975 cell line bearing T790M mutation might result from elevated p-Akt and the deregulation of apoptosis cascade. The reasons for apoptosis evation can be divided as follows: i) overexpression of anti-apoptotic protein, such as Bcl-2, Bcl-xl, ii) lack of pro-apoptotic protein, such as Bax, Bad, iii) higher expression of surviving genes, for instance, survivin (37). In our experiments, we examined protein level of apoptosis-related proteins and found there was no distinction in Bax protein expression but a distinguishable difference in $\mathrm{Bcl}-2$ protein expression between two cell lines of NSCLC on the basis of obvious discrepancy in sensitivity to 
gefitinib. Our results were in line with previous findings that Bcl-2 protein expression level would increase when cells were treated with certain chemotherapy drugs and became resistant $(38,39)$. The finding was unexpected since $\mathrm{Bcl}-2$ is rarely expressed in NSCLC, unlike the level of Bcl-2 that is abundant in small cell lung cancer (SCLC) (40). We thus hypothesized that substantial Bcl-2 might be related to gefitinib resistance. Therapy targeted at anti-apoptotic protein from Bcl-2 family might reactivate the apoptosis signaling pathway.

The function of Bcl-2 in Bcl-2 overexpressing tumors such as lymphomas and SCLC have been explored. Clinically, chronic lymphocytic leukemia (CLL) is suitable for targeting by imatinib. A great body of basic experiments investigated the possible mechanism of acquired drug-resistance to find new approaches to resensitize the malignant cells to the treatment. Bcl-2 overexpression is a hallmark in CLL refractory patients (41). Phase I study of Navitoclax (ABT-263) or pan Bcl-2 family inhibitor Obatoclax in CLL patients with relapsed disease showed that Bcl-2 could be a valid therapeutic target $(41,42)$. Similar phase I clinical studies had been done in SCLC, the Bcl-2 family genes were greatly amplified and had achieved good results with acceptable adverse effect $(43,44)$. Similar results were confirmed in hepatocarcinoma cells resistant to LCL161 with a combination of Bcl-2 siRNA and LCL161 (45). In our study, data confirmed that the cytotoxic and pro-apoptosis effects in $\mathrm{H} 1975$ cell line induced by Bcl-2 siRNA alone could be enhanced when administered together with gefitinib thus reversing acquired EGFR-TKI resistance.

Our data agree with that reported by Fan and co-workers who demonstrated that the established early TKI resistant cell lines exhibited dependence on activation of $\mathrm{Bcl}-2$ / Bcl-xl signaling (46). They also found that when using Bcl-2 homology domain 3 mimetic agents ABT-737 could eradicate the tumor cells evading TKI drugs. Contrary to us, they found that mere Bcl-2 siRNA could not induce dramatic reduction in the early TKI-resistant tumor cells. Dual knockdown of Bcl-2/Bcl-xl manifested more advantageously in cytotoxicity comparing to single intervention. We propose the disparity might lie in the different cell models, the very 'early' molecular events found in their study were probably not the same with the protein changes discovered by us in established cell lines.

However, the precise mechanism for increased Bcl-2 expression in H1975 cell line is still unclear. One potential mechanism may be that micro-RNAs involved in regulating target genes include Bcl-2 (47-49). A panel of miRNAs (miR-16, miR-143) suppressed by estradiol (E2) dramatically induce Bcl-2 expression in breast cancer cells (47). Wang et al determined that miR-181d may act as a glioma suppressor by targeting K-ras and Bcl-2 (48). Similar observation was reported where miR-136 was downregulated in human glioma and lost the capacity to repress anti-apoptotic genes, AEG-1 and Bcl-2 (49). In addition, hepatitis B virus pre-S2 large mutant surface antigen (HBV pre-S2D) increased Bcl-2 expression in hepatocellular carcinoma cells (50). Therefore, further examination of the specific mechanism that Bcl-2 overexpressed in H1975 lung cancer cells are undertaken in our laboratory.
Only a few studies have focused on combining Bcl-2 siRNA with targeted therapy except in CLL. In this study, we confirmed the effect of Bcl-2 siRNA on EGFR-TKI acquired resistant H1975 lung cancer cell line. Although we have verified that Bcl-2 was vital for cell proliferation, anti-apoptotic, inducing acquired drug resistance in H1975 cell line, there are still certain limitations which need further clarification. However, notwithstanding the limitation, this study does suggest the effect of Bcl-2 siRNA on sensitizing TKI acquired resistant cell line to gefitinib.

In conclusion, endogenous level of Bcl-2 can predict the cell sensitivity to EGFR-TKI gefitinib and is an important determinant in gefitinib resistance. Our in vitro study suggested that knockdown the expression of Bcl-2 by siRNA can reverse drug resistance to gefitinib in $\mathrm{H} 1975$ lung cancer cell line harboring T790M mutation. Thus, these findings provide a new concept for the development of novel therapeutic approaches in the treatment of refractory and relapsed patients who are no longer sensitive to EGFR-TKI drugs. Combination of common-used therapy with one targeted to downstream blocking may raise new hope for cure.

\section{Acknowledgements}

This study was supported by National Natural Science Foundation of China (no. 81172187) and in part by grant from the Wujieping Foundation, China (no. 320.6720.10010).

\section{References}

1. Siegel R, Naishadham D and Jemal A: Cancer statistics, 2012. CA Cancer J Clin 62: 10-29, 2012.

2. Tracy S, Mukohara T, Hansen M, Meyerson M, Johnson BE and Janne PA: Gefitinib induces apoptosis in the EGFRL858R non-small-cell lung cancer cell line H3255. Cancer Res 64: 7241-7244, 2004.

3. Lynch TJ, Bell DW, Sordella R, et al: Activating mutations in the epidermal growth factor receptor underlying responsiveness of non-small-cell lung cancer to gefitinib. N Engl J Med 350: 2129-2139, 2004

4. Pao W, Miller V, Zakowski M, et al: EGF receptor gene mutations are common in lung cancers from 'never smokers' and are associated with sensitivity of tumors to gefitinib and erlotinib. Proc Natl Acad Sci USA 101: 13306-13311, 2004.

5. Sequist LV, Martins RG, Spigel D, et al: First-line gefitinib in patients with advanced non-small-cell lung cancer harboring somatic EGFR mutations. J Clin Oncol 26: 2442-2449, 2008.

6. Kobayashi S, Boggon TJ, Dayaram T, et al: EGFR mutation and resistance of non-small-cell lung cancer to gefitinib. N Engl J Med 352: 786-792, 2005.

7. Pao W, Miller VA, Politi KA, et al: Acquired resistance of lung adenocarcinomas to gefitinib or erlotinib is associated with a second mutation in the EGFR kinase domain. PLoS Med 2: e73, 2005.

8. Engelman JA, Zejnullahu K, Mitsudomi T, et al: MET amplification leads to gefitinib resistance in lung cancer by activating ERBB3 signaling. Science 316: 1039-1043, 2007.

9. Bianco R, Shin I, Ritter CA, et al: Loss of PTEN/MMAC1/ TEP in EGF receptor-expressing tumor cells counteracts the antitumor action of EGFR tyrosine kinase inhibitors. Oncogene 22: 2812-2822, 2003.

10. Sos ML, Koker M, Weir BA, et al: PTEN loss contributes to erlotinib resistance in EGFR-mutant lung cancer by activation of Akt and EGFR. Cancer Res 69: 3256-3261, 2009.

11. Guix M, Faber AC, Wang SE, et al: Acquired resistance to EGFR tyrosine kinase inhibitors in cancer cells is mediated by loss of IGF-binding proteins. J Clin Invest 118: 2609-2619, 2008.

12. Jain A, Tindell CA, Laux I, et al: Epithelial membrane protein-1 is a biomarker of gefitinib resistance. Proc Natl Acad Sci USA 102: 11858-11863, 2005. 
13. Uchida A, Hirano S, Kitao H, et al: Activation of downstream epidermal growth factor receptor (EGFR) signaling provides gefitinib-resistance in cells carrying EGFR mutation. Cancer Sci 98: 357-363, 2007.

14. Shaw AT, Yeap BY, Mino-Kenudson M, et al: Clinical features and outcome of patients with non-small-cell lung cancer who harbor EML4-ALK. J Clin Oncol 27: 4247-4253, 2009.

15. Tang JM, He QY, Guo RX and Chang XJ: Phosphorylated Akt overexpression and loss of PTEN expression in non-small cell lung cancer confers poor prognosis. Lung Cancer J Iaslc 51: 181-191, 2006

16. Scrima M, De Marco C, Fabiani F, et al: Signaling networks associated with AKT activation in non-small cell lung cancer (NSCLC): new insights on the role of phosphatydil-inositol-3 kinase. PLoS One 7: e30427, 2012.

17. Brognard J, Clark AS, Ni Y and Dennis PA: Akt/protein kinase B is constitutively active in non-small cell lung cancer cells and promotes cellular survival and resistance to chemotherapy and radiation. Cancer Res 61: 3986-3997, 2001.

18. Janmaat ML, Kruyt FA, Rodriguez JA and Giaccone G: Response to epidermal growth factor receptor inhibitors in non-small cell lung cancer cells: limited antiproliferative effects and absence of apoptosis associated with persistent activity of extracellular signal-regulated kinase or Akt kinase pathways. Clin Cancer Res 9: 2316-2326, 2003

19. Yao M, Zhang W, Zhang Q, et al: Overexpression of MUC1 enhances proangiogenic activity of non-small-cell lung cancer cells through activation of Akt and extracellular signal-regulated kinase pathways. Lung 189: 453-460, 2011.

20. Motzer RJ, Escudier B, Oudard S, et al: Efficacy of everolimus in advanced renal cell carcinoma: a double-blind, randomised, placebo-controlled phase III trial. Lancet 372: 449-456, 2008.

21. Nakachi I, Naoki K, Soejima K, et al: The combination of multiple receptor tyrosine kinase inhibitor and mammalian target of rapamycin inhibitor overcomes erlotinib resistance in lung cancer cell lines through c-Met inhibition. Mol Cancer Res 8: 1142-1151,2010.

22. Price KA, Azzoli CG, Krug LM, et al: Phase II trial of gefitinib and everolimus in advanced non-small cell lung cancer. J Thorac Oncol 5: 1623-1629, 2010

23. Brown I, Shalli K, McDonald SL, et al: Reduced expression of p27 is a novel mechanism of docetaxel resistance in breast cancer cells. Breast Cancer Res 6: R601-R607, 2004.

24. Costa DB, Halmos B, Kumar A, et al: BIM mediates EGFR tyrosine kinase inhibitor-induced apoptosis in lung cancers with oncogenic EGFR mutations. PLoS Med 4: 1669-1679, $1680,2007$.

25. Lima RT, Martins LM, Guimaraes JE, Sambade C and Vasconcelos MH: Specific downregulation of bcl-2 and xIAP by RNAi enhances the effects of chemotherapeutic agents in MCF-7 human breast cancer cells. Cancer Gene Ther 11: 309-316, 2004

26. Tumilasci VF, Oliere S, Nguyen TL, Shamy A, Bell J and Hiscott J: Targeting the apoptotic pathway with BCL-2 inhibitors sensitizes primary chronic lymphocytic leukemia cells to vesicular stomatitis virus-induced oncolysis. J Virol 82: 8487-8499, 2008.

27. Aoki Y, Cioca DP, Oidaira H, Kamiya J and Kiyosawa K: RNA interference may be more potent than antisense RNA in human cancer cell lines. Clin Exp Pharmacol Physiol 30 : 96-102, 2003

28. Witta SE, Gemmill RM, Hirsch FR, et al: Restoring E-cadherin expression increases sensitivity to epidermal growth factor receptor inhibitors in lung cancer cell lines. Cancer Res 66 944-950, 2006.

29. Baselga J, Rischin D, Ranson M, et al: Phase I safety, pharmacokinetic, and pharmacodynamic trial of ZD1839, a selective oral epidermal growth factor receptor tyrosine kinase inhibitor, in patients with five selected solid tumor types. J Clin Oncol 20 : 4292-4302, 2002.

30. Bursztajn S, Feng JJ, Berman SA and Nanda A: Poly (ADP-ribose) polymerase induction is an early signal of apoptosis in human neuroblastoma. Brain Res Mol Brain Res 76: 363-376, 2000.

31. Wu XH, Lu Y, Fang YW and Jiang YX: The polyamidoaminemediated inhibition of bcl-2 by small hairpin RNA to induce apoptosis in human lens epithelial cells. Mol Vis 18: 74-80, 2012
32. George J, Banik NL and Ray SK: Combination of taxol and Bcl-2 siRNA induces apoptosis in human glioblastoma cells and inhibits invasion, angiogenesis and tumour growth. J Cell Mol Med 13: 4205-4218, 2009.

33. Dudek H, Datta SR, Franke TF, et al: Regulation of neuronal survival by the serine-threonine protein kinase Akt. Science 275: 661-665, 1997.

34. Janmaat ML, Rodriguez JA, Gallegos-Ruiz M, Kruyt FA and Giaccone G: Enhanced cytotoxicity induced by gefitinib and specific inhibitors of the Ras or phosphatidyl inositol-3 kinase pathways in non-small cell lung cancer cells. Int J Cancer 118: 209-214, 2006

35. Morgillo F, D'Aiuto E, Troiani T, et al: Antitumor activity of bortezomib in human cancer cells with acquired resistance to anti-epidermal growth factor receptor tyrosine kinase inhibitors. Lung Cancer J Iaslc 71: 283-290, 2011.

36. Zitzmann K, Ruden J, Brand S, et al: Compensatory activation of Akt in response to mTOR and Raf inhibitors - a rationale for dual-targeted therapy approaches in neuroendocrine tumor disease. Cancer Lett 295: 100-109, 2010.

37. Han SW and Roman J: Targeting apoptotic signaling pathways in human lung cancer. Curr Cancer Drug Targets 10: 566-574, 2010.

38. Losert D, Pratscher B, Soutschek J, et al: Bcl-2 downregulation sensitizes nonsmall cell lung cancer cells to cisplatin, but not to docetaxel. Anticancer Drugs 18: 755-761, 2007.

39. Yoo SH, Yoon YG, Lee JS, et al: Etoposide induces a mixed type of programmed cell death and overcomes the resistance conferred by Bcl-2 in Hep3B hepatoma cells. Int J Oncol 41: 1443-1454, 2012

40. Lawson MH, Cummings NM, Rassl DM, et al: Bcl-2 and beta1integrin predict survival in a tissue microarray of small cell lung cancer. Br J Cancer 103: 1710-1715, 2010.

41. Roberts AW, Seymour JF, Brown JR, et al: Substantial susceptibility of chronic lymphocytic leukemia to BCL2 inhibition: results of a phase I study of navitoclax in patients with relapsed or refractory disease. J Clin Oncol 30: 488-496, 2012.

42. Schimmer AD, O'Brien S, Kantarjian H, et al: A phase I study of the pan bcl-2 family inhibitor obatoclax mesylate in patients with advanced hematologic malignancies. Clin Cancer Res 14: 8295-8301, 2008 .

43. Gandhi L, Camidge DR, Ribeiro DOM, et al: Phase I study of Navitoclax (ABT-263), a novel Bcl-2 family inhibitor, in patients with small-cell lung cancer and other solid tumors. J Clin Oncol 29: 909-916, 2011

44. Chiappori AA, Schreeder MT, Moezi MM, et al: A phase I trial of pan-Bcl-2 antagonist obatoclax administered as a 3-h or a 24-h infusion in combination with carboplatin and etoposide in patients with extensive-stage small cell lung cancer. Br J Cancer 106: 839-845, 2012.

45 Chen KF, Lin JP, Shiau CW, et al: Inhibition of Bcl-2 improves effect of LCL161, a SMAC mimetic, in hepatocellular carcinoma cells. Biochem Pharmacol 84: 268-277, 2012.

46. Fan W, Tang Z, Yin L, et al: MET-independent lung cancer cells evading EGFR kinase inhibitors are therapeutically susceptible to BH3 mimetic agents. Cancer Res 71: 4494-4505, 2011.

47. Yu X, Zhang X, Dhakal IB, Beggs M, Kadlubar S and Luo D: Induction of cell proliferation and survival genes by estradiolrepressed microRNAs in breast cancer cells. BMC Cancer 12: 29 , 2012.

48. Wang XF, Shi ZM, Wang XR, et al: MiR-181d acts as a tumor suppressor in glioma by targeting K-ras and Bcl-2. J Cancer Res Clin Oncol 138: 573-584, 2012.

49. Yang Y, Wu J, Guan H, et al: MiR-136 promotes apoptosis of glioma cells by targeting AEG-1 and Bcl-2. FEBS Lett 586: 3608-3612, 2012

50. Hung JH, Teng YN, Wang LH, et al: Induction of Bcl-2 expression by hepatitis $\mathrm{B}$ virus pre-S2 mutant large surface protein resistance to 5-fluorouracil treatment in Huh-7 cells. PLoS One 6: e28977, 2011. 